\begin{tabular}{|c|c|c|}
\hline & Int.J.Curr.Microbiol.App.Sci (2021) 10(10): 193-198 & \\
\hline & $\begin{array}{l}\text { International Journal of Current Microbiology and Applied Sciences } \\
\text { ISSN: 2319-7706 Volume } 10 \text { Number } \mathbf{1 0}(\mathbf{2 0 2 1 )} \\
\text { Journal homepage: http://www.ijcmas.com }\end{array}$ & 30 \\
\hline $\begin{array}{l}\text { EXCELLENT } \\
\text { PUBLISHERS }\end{array}$ & & \\
\hline
\end{tabular}

Case Study https://doi.org/10.20546/ijcmas.2021.1010.022

\title{
Incidental Laboratory Identification of Brucella melitensis in a case with Pancytopenia
}

\author{
Mahalakshmi Kumaresan ${ }^{1}$, Lakshmi Shanmugam ${ }^{1}$, Ketan Priyadarshi, ${ }^{1}$, \\ Mahathi Gopalakrishnan ${ }^{2}$, Tamilarasu Kadhiravan ${ }^{2}$ and Apurba Sankar Sastry ${ }^{1 *}$ \\ ${ }^{I}$ Department of Microbiology, ${ }^{2}$ Department of General Medicine, JIPMER, Puducherry, India \\ *Corresponding author
}

A B S T R A C T

Keywords

Brucellosis, occupation, dairy, bone marrow suppression, pancytopenia

Article Info

Accepted:

10 September 2021 Available Online: 10 October 2021
Brucellosis is a bacterial zoonosis usually associated with exposure to infected animals or their products. Although a significant public health problem in India, exact prevalence and distribution are unknown owing to the imprecision of diagnosis and inadequacy of reporting and surveillance. Although the febrile illness is common, its manifestations are highly variable. Bone marrow suppression and consequent pancytopenia have been rarely reported. We present a case of 50 years old female diagnosed with human brucellosis associated with pancytopenia and non-specific clinical presentation, that was diagnosed incidentally on blood and bonemarrow culture. This was confirmed by serological tests like the standard agglutination test. Culture isolation using automated blood culture (e.g. BacT/ALERT), followed by identification using automated identification systems (e.g. MALDI-TOF and VITEK-2) help to reach accurate and timely diagnosis aiding in the management of the patient.

\section{Introduction}

\section{Case Report}

A 50 years old non-diabetic non-hypertensive female, milkmaid by occupation from southern India presented to the medicine outpatient department with complaints of vague non-localizing lower backache, easy fatiguability, and generalized weakness for the past one month along with intermittent nonprojectile vomiting for seven days. She also complained of unexplained weight loss and anorexia for the last month.

On clinical examination, she had moderate pallor, with no signs of icterus, pedal edema, lymphadenopathy, clubbing, or cyanosis. She also had mild hepato-splenomegaly. During the initial course of stay in the hospital, the 
patient developed spikes of mild grade fevernot associated with chills, rigor, or any rashes. On routine hemogram, the patient was diagnosed to have normocytic anaemia $(\mathrm{Hb}$ $8.9 \mathrm{gm} / \mathrm{dL}$ ), thrombocytopenia (platelet count of $92,000 / \mu \mathrm{L}$ ) as well as leucopenia (total leucocyte count of $3000 / \mu \mathrm{L}$ ).

Because of the fever spikes, a blood culture specimen was sent to microbiology laboratory in BacT/ALERT automated adult aerobic blood culture bottle, which was immediately incubated in VirtuoBacT/ALERT system (bioMerieux) at $37^{\circ} \mathrm{C}$. The bottle flagged positive after 63 hours of incubation. Gram staining was performed directly from flagged broth, which showed short gram-negative coccobacilli. Subculture from the bottle was performed on 5\% sheep blood agar (SBA), chocolate agar (CA), and MacConkey agar (MAC), and incubated aerobically at $37^{\circ} \mathrm{C}$. Faint colonies were visible after 20 - 22 hours of aerobic incubation. Gram staining from colony showed gram-negative coccobacilli (approx. $1.5 \mu \mathrm{m} \times 1 \mu \mathrm{m}$ ), which was catalase and oxidase test positive. Automated identification was performed using Matrixassisted laser desorption/ionization-time of flight mass spectrometry (VITEK-MS), which identified it as Brucella species. Identification was subsequently confirmed using conventional phenotypic tests. Brucella differential stain (Stamp's modification of Ziehl - Neelsen staining) was performed in which organism appeared reddish-pink coccobacilli. Urea was rapidly hydrolyzed within 20 minutes of aerobic incubation. Growth was enhanced on incubation in capnophilic $\left(5-10 \% \quad \mathrm{CO}_{2}\right)$ incubation. Species iidentification was made by using the VITEK-2 automated system using the gramnegative identification card, which was identified as Brucella melitensis. Subsequently, serology by standard agglutination test (SAT) was also performed on the patient's serum to detect the presence of agglutinating antibodies against Brucella, which also turned out reactive with a titer of $1: 320$.

Paired blood culture was repeated after five days from initial blood culture which flagged positively with time to positivity of 66 hours and isolate was again identified as Brucella melitensis. Because of existing pancytopenia, diagnostic bone marrow aspiration was performed and the bone marrow sample was sent for aerobic culture directly in paired BacT/ALERT pediatric blood culture bottles. The bottles flagged positively at 55 hours and 60 hours of incubation respectively and yielded subsequently the same organism, Brucella melitensis. Bone marrow cytology revealed hypocellular patches, with suppressed leucopoiesis and erythroid hyperplasia.

Following laboratory confirmation, detailed history was evaluated for zoonotic exposure to the patient. The patient was a milkmaid by profession; frequently involved handling of cattle. She also gave an occasional history of intake of unpasteurized raw cow milk. In addition, she revealed that she had an episode of oral ulcers for the past 15 days, for which she has taken goat milk as a local remedy a few days back.

Two-dimensional echocardiography (ECHO) was performed to rule out infective endocarditis as vegetations could serve as a niche for this organism but ECHO does not reveal any vegetations. Because of persistent backache, an X-ray of the sacroiliac joint was performed to rule out sacroiliitis, which was found to be normal. No other features suggestive of focal brucellosis or complicated brucellosis could be elicited.

On reporting of Brucella from blood culture, the patient was started on oral doxycycline 200 mg twice daily and injectable streptomycin 
1gram intramuscularly. She improved clinically after seven days of antimicrobial therapy and was subsequently discharged on injectable streptomycin and oral doxycycline for 3 months. Informed consent was obtained subsequently from the patient during the stay in the hospital.

\section{Results and Discussion}

Brucellosis is a bacterial zoonosis and is usually associated with occupational or domestic exposure to infected animals or their products like ingestion of unpasteurized raw milk(1).Though it is a significant public health problem in India, the exact prevalence and distribution are unknown owing to the imprecision of diagnosis and the inadequacy of reporting and surveillance systems.

Brucella is an obligately aerobic, catalasepositive, oxidase-positive, fastidious, small intracellular gram-negative coccobacillus. Brucella melitensis accounts for the major proportion of human infections(2,3). Brucellosis is a multisystem infection that may present with a broad spectrum of clinical presentations with the most common being acute febrile illness and musculoskeletal symptoms. It tends to involve reticuloendothelial cells. Its clinical manifestations are highly variable, and definitive symptoms indicative of the diagnosis may be lacking. Bone marrow involvement can lead to hematological complications such as anemia, leukopenia, or pancytopenia, and arerarely reported $(4,5,6)$. Thus even on the smallest amount of clinical suspicion, the diagnosis must be attempted using bacteriologic and/or serologic tests. Brucella species have been reported in patients presenting with autoimmune hemolytic anemia (2), febrile neutropenia (7), immune thrombocytopenic purpura (8)' disseminated intravascular coagulation (9), myelodysplastic syndrome (4). Brucella infection is known to induce several hematological abnormalities already well described in the above-mentioned literature. Leukopenia is more common, occurring in 16 to $25 \%$ of patients (5) whereas the frequency of pancytopenia ranges from 3 to $20 \%$ (6) Our patient presents with pancytopenia similar to several other studies (3-7). Pancytopenia associated with brucellosis is attributed to hypersplenism, hemophagocytosis, and granulomatous lesions of the bone marrow, which is usually hypercellular. Bone marrow hypoplasia is rarely reported. (3) This is probably because the organisms exert a direct inhibitory effect on marrow cells or they induce lymphocytes to release mediators that inhibit hematopoiesis(3).

Brucella is a facultative intracellular bacterium, and disseminate through the hematogenous routein the body (10). There is a strong predilection for organs of the reticuloendothelial system consequently, brucellosis often presents with hematologic abnormalities $(7,10)$.

In this study performed a literature review on PubMed search database using the search term "Brucella" and "pancytopenia". Around 40 literatures were found of brucellosis presenting as pancytopenia. On literature review, most common age group reported with brucellosis and cytopenia were children and adolescents (11-12). Adults have been reported sporadically (4-7), as in our case.

We could not determine initially whether the patient had pancytopenia secondary to brucellosis, or the brucellosis occurred in preexisting pancytopenia. 
Fig.1 Gram negative coccobacilli resembling Brucella species at 100X magnification in Gram stain from flagged blood culture bottle

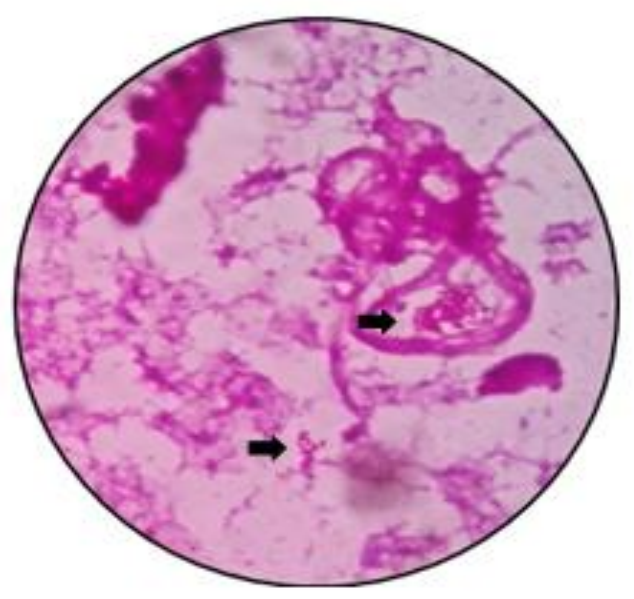

Fig.2 Gram negative coccobacilli resembling Brucella species at 100X magnification in Gram stain from colony

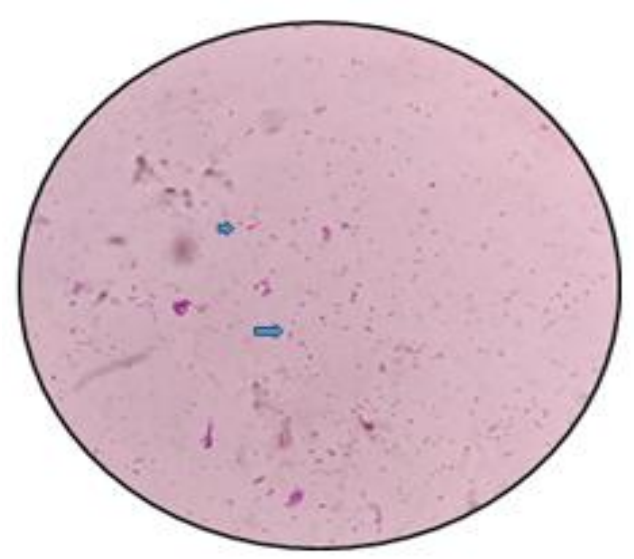

Fig.3 Reddish pink acid-fast coccobacilli resembling Brucella species at 100X magnification in Stamp's modified Ziehl-Neelsen staining from colony

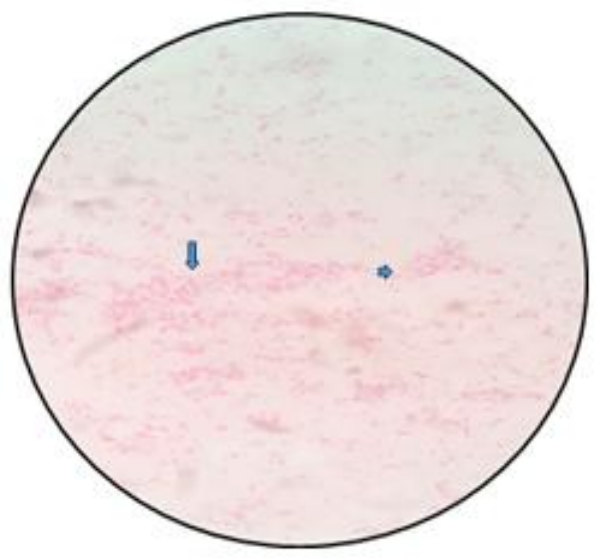


Fig.4 Small non-pigmented grey colonies of Brucella species on Chocolate agar after 48 hours of capnophilic incubation

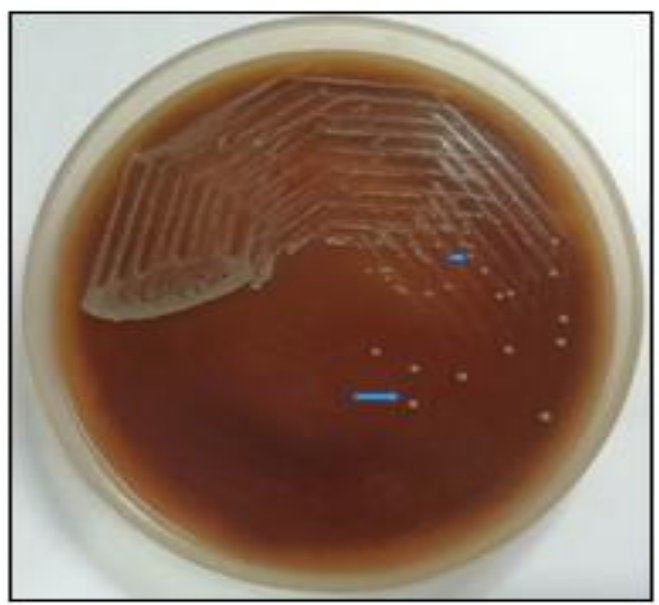

However, isolation of Brucella from bonemarrow culture and the remarkable clinical response after starting targeted therapy for the infection support the hypothesis that Brucella was the cause of the pancytopenia. Simultaneous isolation of Brucella from blood and bone-marrow culture has also been reported from other studies (13).

The possible source of the infection was traced back to one of three zoonotic exposure i) milkmaid by profession; frequently involved handling of cattle; ii) history of intake of unpasteurized raw cow milk; iii) history of goat milk as a local remedy a few days back, as found in other studies (12). These findings suggest the potential source of exposure to this zoonotic pathogen in this patient with bone marrow suppression and pancytopenia.

Bone marrow hypoplasia is rarely reported and should be kept in mind in the etiology of aplastic anemia in a country where brucellosis is frequently encountered. Pancytopenia with hypocellular bone marrow although rare, may complicate the course of Brucella infection. We conclude that it is always important to obtain a comprehensive patient history and consider brucellosis as one of the broad differential diagnoses for any patient with a vague constellation of symptoms of back pain, easy fatiguability, and generalized weakness and subsequently warrant automated blood cultures.

\section{Conflicts of Interest}

The authors declare that there are no conflicts of interest regarding the publication of this article.

\section{References}

1. Ataman Hatipoglu C, Yetkin A, TuncerErtem G, Tulek N. Unusual clinical presentations of brucellosis. Scand J Infect Dis. 2004 Sep 1;36(9):695-8.

2. Meena D S, Sonwal V S, Rohila A K, Meena V. Acute Brucellosis Presenting as an Autoimmune Hemolytic Anemia. Case reports in infectious diseases. 2018 Oct $25 ; 2018$.

3. Yildirmak Y, Palanduz A, Telhan L, Arapoglu M, Kayaalp N. Bone marrow hypoplasia during Brucella infection. $J$ PediatrHematolOncol. 2003 Jan 1;25(1):63-4.

4. Li J J, Sheng Z K, Tu S, Bi S, Shen X M, 
Sheng J F. Acute brucellosis with myelodysplastic syndrome presenting as pancytopenia and fever of unknown origin. Medical Principles and Practice. 2012;21(2):183-5.

5. Lynch E C, Mckechnie J G, Alfrey J R C P. Brucellosis with pancytopenia. Ann Intern Med. 1968 Aug 1;69(2):319-22.

6. Starakis I, Polyzogopoulou E, Siagris D, Mazokopakis E, Gogos C A. Unusual manifestation of brucellosis: liver abscess and pancytopenia caused by Brucella melitensis. Eur. J. Gastroenterol. Hepatol. 2008 Apr 1;20(4):349-52.

7. Chang C, Beutler B D, Ulanja M B, Uche C, Zdrnja M. Brucellosis Presenting with Febrile Pancytopenia: An Atypical Presentation of a Common Disease and Review of Brucellosis. Case Reports in Infectious Diseases. 2021 Jan 4;2021.

8. Makis A, Perogiannaki A, Chaliasos N. Severe thrombocytopenic purpura in a child with brucellosis: case presentation and review of the literature. Case reports in infectious diseases. 2017 Jan 3;2017.
9. Turunc T, Demiroglu Y Z, Kizilkilic E, Aliskan H, Boga C, Arslan H. A case of disseminated intravascular coagulation caused by Brucella melitensis. J Thromb Thrombolysis. 2008 Aug;26(1):71-3.

10. Mantur B G, Amarnath S K. Brucellosis in India-a review. J. Biosci. 2008 Nov 1;33(4):539-47

11. Justman N, Fruchtman Y, Greenberg D, Ben-Shimol S. Hematologic Manifestations of Brucellosis in Children. Pediatr Infect Dis J. 2018 Jun;37(6):586591. doi: 10.1097/ INF.0000000000001900. PMID: 29329166.

12. El-Koumi M A Md, Afify M Md, AlZahrani S H Md. A prospective study of brucellosis in children: relative frequency of pancytopenia. Iran J Pediatr. 2014 Apr;24(2):155-60. PMID: 25535533; PMCID: PMC4268834.

13. Hamilton $\mathrm{P} K \mathrm{~K}$. The bone marrow in brucellosis. American journal of clinical pathology. 1954;24(5):580-87.

\section{How to cite this article:}

Mahalakshmi Kumaresan, Lakshmi Shanmugam, Ketan Priyadarshi, Mahathi Gopalakrishnan, Tamilarasu Kadhiravan and Apurba Sankar Sastry. 2021. Incidental Laboratory Identification of Brucella melitensis in a case With Pancytopenia. Int.J.Curr.Microbiol.App.Sci. 10(10): 193198. doi: https://doi.org/10.20546/ijcmas.2021.1010.022 